\title{
Actualización: \\ Educación para la salud basada en la comunidad (segunda entrega)
}

Community based health education

Nanci Giraudo y Javier Chiarpenello

\begin{abstract}
Resumen
En los últimos siglos la medicina sufrió importantes transformaciones por las cuales podemos hoy distinguir dos vertientes: la medicina clínica y la medicina social. Esta última centró su mirada en un entorno ambiental y social, implicando un salto cualitativo importante y remarcando la importancia de la educación y la promoción de la salud como una práctica más del médico.

En una entrega anterior los autores desarrollan conceptos importantes, como ser: generalidades, principios y objetivos de la educación para la salud; fundamentos de las metodologías y técnicas participativas, estrategias para optimizar la efectividad de una intervención comunitaria, así como los principios de la organización comunitaria. En esta segunda y última entrega los autores completan la actualización desarrollando temas como: planificación, diagnostico de situación y selección de estrategias educativas; el diagnóstico de los procesos administrativos; la evaluación global del proceso implementado, así como también las condiciones para el éxito de una intervención o programa educativo.
\end{abstract}

\begin{abstract}
In recent centuries, medicine underwent major transformations allowing the identification of two branches: clinical medicine and social medicine. The latter focused its attention on environmental and social aspects, involving a major leap forward and highlighting the importance of education and health promotion as and additional medical value.

In a previous issue the authors developed important concepts, such as: health education general principles and objectives; participatory methodologies and techniques essentials; strategies to optimize the effectiveness of community interventions, and community organizational principles. In this second and final issue authors will expand on related concepts such as: planning, situational diagnosis, educational strategies selection, diagnosis of administrative processes, the overall assessment of implemented processes, as well as the needed conditions for reaching success on educational interventions.
\end{abstract}

Palabras clave: educación para la salud, salud comunitaria, promoción de la salud. Key words: health education, health community, health promotion.

Giraudo N, Chiarpenello J. Educación para la salud basada en la comunidad (segunda entrega). Evid Act Pract Ambul. Ene-Mar 2012;15(1):21-25.

\section{Planificación}

Toda actividad de promoción y prevención a implementar en la comunidad requiere de una planificación previa, cuyo punto fundamental es el diagnóstico de necesidad o de situación, con el objetivo de satisfacer las demandas reales de una comunidad, cosa que no ocurre con frecuencia.

Las preguntas que siempre se deben tener presentes a la hora de una intervención comunitaria son las siguientes: ¿Cuál es la necesidad? ¿Cómo se va a resolver el problema? ¿Cuál es la base o sustento teórico del problema? ¿Qué propone la gente? En este sentido es importante pensar que siempre las soluciones están en la propia comunidad, y no que somos nosotros los que tenemos las "soluciones".

\section{Diagnóstico de necesidad}

Según, un trabajo realizado por Kerr White en 1961, la mayoría de las personas que experimentan un problema de salud no consulta al médico. Por lo tanto, la información sobre la distribución de los problemas de salud de una comunidad no puede ni debe basarse sólo en la experiencia de médicos trabajando en hospitales o consultorios.

En el área de la salud, una necesidad puede ser definida como la capacidad que tiene un individuo o una población de beneficiarse de una intervención ó tratamiento que se encuentren disponibles con fines preventivos, curativos o de rehabilitación. Existen muchas definiciones de necesidad, siendo la más utilizada a la hora de planificar en salud, la de Bradshaw y col., quienes distinguieron cuatro tipos que se resumen en el cuadro 4.

No siempre hay coincidencia en cuanto a las necesidades definidas por el equipo de salud y las necesidades definidas por la gente, situación álgida y a tener en cuenta a la hora de realizar una intervención en una comunidad (ver figura 2). Esta realidad hace necesario compartir con la gente los diferentes puntos de vistas, reflexionar sobre las condiciones y características de sus procesos de salud-enfermedad y sus manifestaciones, así como discutir las formas de llegar a comprender los modos posibles de encarar con eficacia, los daños emergentes de esos procesos. Es importante recordar que muchas, por no decir la mayoría de las soluciones, surgen en el seno de la comunidad y que no existen recetas mágicas.

Por lo tanto, es importante tener en cuenta que los tipos de problemas sociales que una comunidad está experimentando o padeciendo son buenos indicadores de su calidad de vida. Para evaluar dicha calidad de vida y para luego intervenir hay que aunar esfuerzos para saber interpretar correctamente cuales son las preocupaciones que presenta la comunidad y conocer los roles de cada uno de los integrantes del grupo pasibles de una intervención educativa.

En este proceso de diagnóstico de necesidad no debemos dejar de identificar los denominados factores no conductuales como los factores económicos, genéticos y ambientales que influyen en la salud y que contribuirán a proporcionar una visión mas amplia de las limitaciones con las que nos podemos encontrar en este proceso de educación para la salud.

Como parte del diagnóstico de necesidad debemos tener en cuenta las características epidemiológicas de la población (distribución de los factores de riesgo y de los problemas de salud) para tener una idea global sobre de las necesidades de los integrantes de la comunidad.

Cuadro 4: los cuatro tipos de necesidad descriptos por Bradshaw y col.

- Necesidad normativa: es la establecida por los expertos (normas y criterios).

- Necesidad sentida: surge cuando los integrantes de una comunidad manifiestan qué quieren.

- Necesidad expresada: equivale a la demanda de servicios de salud por parte de la comunidad.

- Necesidad comparativa: consiste en evaluar los resultados de los servicios de salud que se ofrecen a una población para definir qué se necesita en una zona comparable.

* Servicio de Medicina Familiar y Comunitaria del Hospital Italiano de Buenos Aires. nanci.giraudo@ hospitalitaliano.org.ar

** Médico Generalista y de Familia. Médico Endocrinólogo. Hospital Centenario de Rosario. jchiarpenello @ hotmail.com 
Figura 2: visualización de problemas o necesidades
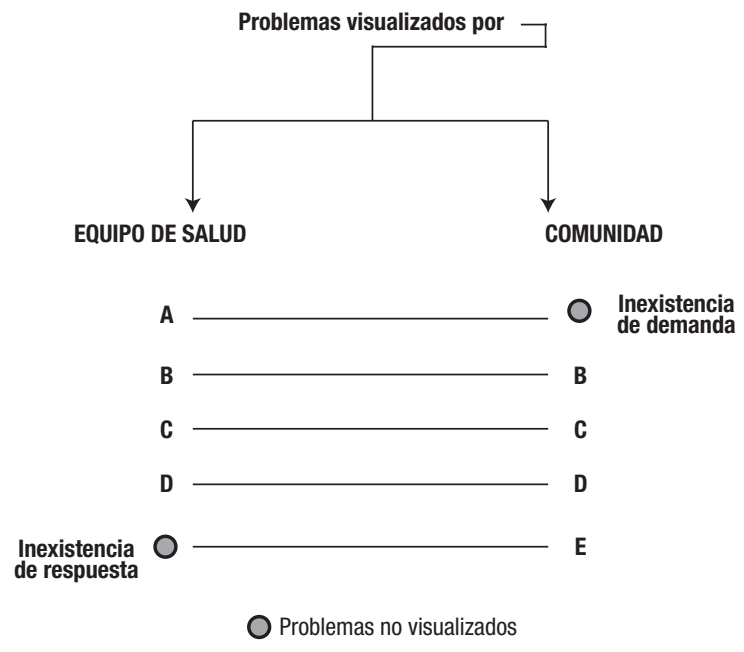

Los problemas B, C y D son identificados tanto por el equipo de salud como por la comunidad. El problema A es identificado sólo por el equipo de salud y el E, sólo por la comunidad. Por lo tanto, los problemas A y E tienen existencia real, pero si ambos -equipo de salud y comunidadno llegan a un acuerdo o redefinición de los mismos, posiblemente no tengan resolución, especialmente el problema $A$, que al no ser percibido como tal, no genera demanda por parte de la comunidad.

\section{Averiguar que hicieron otros antes}

Una vez definida la necesidad o identificado el problema a trabajar, el equipo de trabajo tiene que plantearse cómo resolver dicha situación, para lo cual consultará la bibliografía y a los expertos para ver si hay antecedentes respecto del modo de abordaje de la problemática en cuestión (sustento teórico de la intervención).

Como se mencionó más arriba, es importante y siempre debemos tener presente lo que la gente del lugar propone como solución del problema identificado. En consecuencia, un programa o proyecto bien instruido que responda a las necesidades reales de un grupo destinatario, debe basarse necesariamente en un análisis correcto y completo de la situación. Esta situación deberá interpretarse conforme al interés y a las actividades de las partes interesadas, que a menudo tienen una visión diferente de esta misma realidad.

Existen diferentes formas de analizar una realidad. Por un lado, los estudios realizados por los expertos, que aportan respuestas a las preguntas planteadas, tal como ellos las han concebido, según normas y criterios. Por otro lado, los representantes de la comunidad u organizaciones implicadas tendrán sus propias percepciones. En consecuencia, una reunión en la que participen los representantes de la comunidad (los principales actores en salud) y los expertos, permitirá llegar a una concepción de lo que se pretende, que sea compartida y aceptada por todos.

Averiguar que harían los protagonistas o receptores

Para que la planificación de un servicio, programa o proyecto responda a las necesidades reales de la comunidad es indispensable analizar en conjunto -preferentemente junto con las diversas partes implicadas- los problemas que se plantean, los objetivos formulados y la posible elección de una estrategia (Ver figura 3).

El enfoque descentralizado o centrado en la gente permite: 1) que la planificación y las decisiones básicas tengan lugar a nivel de la comunidad y 2) servir no como cuerpo dirigente sino como centro de comunicaciones, asesoría, de apoyo y de provisión de materiales.

Una intervención, un programa o un proyecto comunitario de promoción y educación para la salud bien instruido y que responda a las necesidades reales de un grupo destinatario debe basarse necesariamente en un análisis correcto y completo de la situación. Esta situación deberá interpretarse conforme al interés y a las actividades de las partes interesadas, que a menudo tienen una visión diferente de esta misma realidad (Ver figura 4).

El determinar beneficios a largo plazo tiene varias ventajas: 1) aumentar la especificidad y perspectiva de la planificación (la claridad de las definiciones de los resultados está directamente relacionada con la probabilidad de seleccionar intervenciones sólidas y apropiadas), 2) brindar a los participantes un panorama de los objetivos planteados, intentando que esto contribuya a un mayor entusiasmo y participación por parte de ellos y 3 ) advertirles sobre los riesgos o beneficios de cursos de acción alternativos.

Se considera que la evaluación de los beneficios es más eficaz cuando se la efectúa antes de realizar la recopilación de datos y se establecen los resultados deseados o esperados, ya que, de esta manera, los planificadores y la comunidad pueden tener una visión más amplia de la intervención.

Figura 3: lógica de una intervención comunitaria

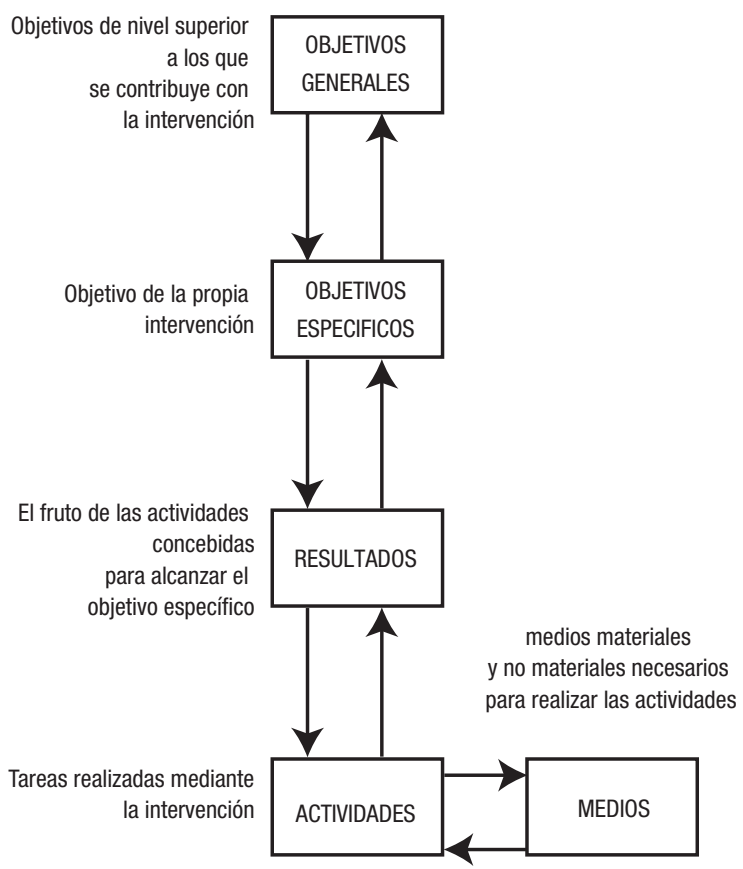


Figura 4: evaluación de un programa comunitario

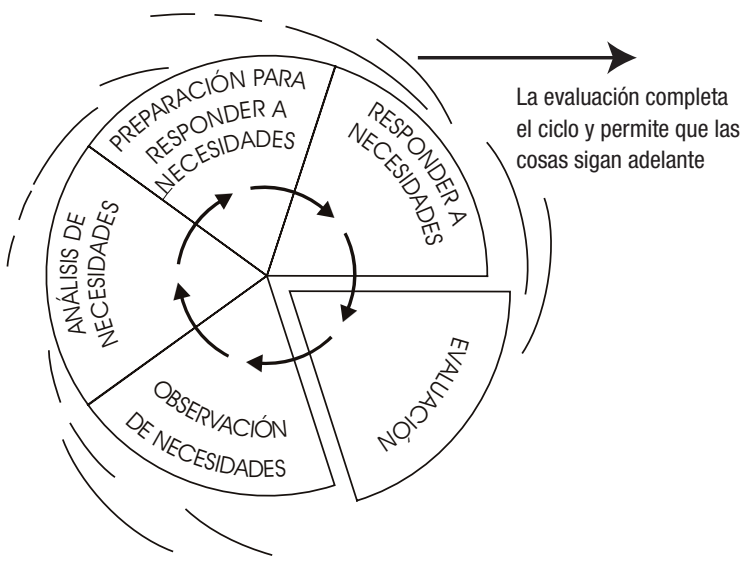

Modificado de Werner D y Bower B. Aprendiendo a Promover la Salud. $4^{\circ}$ reimpresión, 1991. Fundación Hesperian y Centro de estudios educativos. Los exámenes y la evalucación como un proceso de aprendizaje. Capítulo 9 p 182.

\section{Selección de una estrategia educativa}

Podríamos definir una estrategia educativa en salud como una combinación de métodos y técnicas que se puede utilizar para intervenir sobre factores predisponentes, facilitadores y reforzadores de algún suceso o situación. Estas estrategias se pueden clasificar en tres categorías:

- Métodos de comunicación: conferencia-debate, asesorías individuales, medios masivos de comunicación (gráficos y audio-visuales).

- Métodos de capacitación: desarrollo de habilidades, modificación de conductas y de actitudes, a través de juegos, debates en grupos, creación de modelos, etc.

- Métodos organizacionales: desarrollo comunitario, acción y planificación social y desarrollo organizacional.

A la hora de elegir, se recomienda seleccionar al menos tres estrategias educativas y asegurarse de que los factores predisponentes, facilitadores y reforzadores reciban la debida atención. Se aconseja que una de las tres estrategias seleccionadas incluya medios audiovisuales u otro medio de comunicación. Es importante no descuidar a ninguno de los tres factores arriba mencionados, los tres son importantes por igual y nos permiten optimizar los recursos, en especial en el mediano y largo plazo.

Es importante tener en cuenta que las estrategias educativas que influyan sobre los factores predisponentes tendrán efectos generalmente a corto plazo, las que lo hagan sobre los factores reforzadores tendrán efecto intermedio y las que influyan sobre los tres factores tendrán un efecto más prolongado. Tener presente esto ayuda a no defraudarnos ante la falta de respuesta a corto plazo y evitar que abandonemos el rumbo de nuestras metas.

A diferencia de aquellas que lo harán sobre los otros dos factores, las estrategias que influyen sobre los factores predisponentes suelen ser más simples y menos costosas.

Ejercitarnos y esforzarnos e invertir tiempo en aprender el desarrollo de estas estrategias redundará en beneficios a largo plazo y en la optimización de tiempos y resultados. Con la puesta en práctica y ejercitación de estas estrategias y con el trabajo interdisciplinario sobre las mismas es como obtendremos los mejores resultados.

\section{Diagnóstico administrativo}

Como parte del proceso de una intervención es importante incluir en su elaboración un apartado de diagnóstico administrativo, identificando factores administrativos y organizacionales que puedan afectar la implementación exitosa del programa.

La meta principal de este diagnóstico es reducir al mínimo los factores que podrían hacer que una intervención o un programa bien concebido no logren un impacto completo y positivo. Para ello es importante considerar las realidades de la organización, de la comunidad y de la práctica profesional, para reducir así los impactos negativos y potenciar los beneficios de la intervención.

Los pasos de este diagnóstico incluyen la evaluación de los recursos disponibles y la correcta asignación de los mismos mediante un presupuesto y un cronograma, así como determinar las relaciones jerárquicas entre el personal y el recurso humano disponible.

\section{Evaluación}

Mucha gente piensa en la evaluación como algo punitorio y como un proceso complicado que se hace durante un tiempo limitado. Sin embargo debería ser todo lo contrario: sencilla y continua. Comúnmente, la evaluación ocurre cuando las personas que participan en una actividad o proyecto discuten sobre como marchan las cosas. Sin embargo, una evaluación periódica y más estructurada debe tener su lugar.

En este sentido, podemos considerar cuatro tipos de evaluaciones, todas partes de un proceso continuo.

\section{Evaluación continua}

Tiene lugar en todo proyecto o actividad. Por ejemplo, a lo largo de un curso de capacitación de promotores de la salud, los capacitadores o instructores y los alumnos pueden preguntarse: a) ¿qué tan efectiva es tal o cuál clase? y b) ¿qué tanto nos prepara esta actividad para resolver un determinado problema en la comunidad?

\section{Evaluación periódica}

Se hace en ciertos momentos durante un proyecto o actividad. Por ejemplo, los instructores, los alumnos y las personas de la comunidad que participan en un programa pueden reunirse de vez en cuando para examinar el progreso del curso y de ciertas actividades. En un programa de un año de duración esta evaluación podría ser mensual.

\section{Evaluación final}

Tiene lugar al final del proyecto o actividad. Por ejemplo, al final de un curso todos los participantes pueden juntarse para evaluar el curso en su totalidad, con sus debilidades y fortalezas. La idea es ver como se podría mejorar para la próxima vez.

\section{Evaluación de seguimiento}

Se basa en los resultados o en el efecto de una actividad después que se ha finalizado con el proyecto o actividad. Por ejemplo, la eficacia de un curso de capacitación de promotores de la salud se comprueba al ver el éxito que tengan los promotores en ayudar a la gente a satisfacer sus necesidades en la comunidad. Sin embargo, no es fácil evaluar el efecto o "impacto" sobre la comunidad ya que, en general, los resultados son a largo plazo.

Este tipo de evaluación se puede basar en: a) la reacción de la comunidad, b) las visitas de seguimiento de los capacitadores, 
c) los indicadores de salud y d) el informe de los promotores acerca de sus logros y dificultades.

Niveles de la evaluación

La evaluación de un programa, de una actividad o de un proyecto -antes, durante y después de su desarrollo- es clave para que se complete un ciclo.

La evaluación siempre es necesaria si queremos seguir adelante, porque nos permite aprender de los éxitos y de los errores, además de ayudarnos a subsanarlos. La figura 4 adaptada de Werner y col. ejemplifica este proceso.

Haciendo referencia a la evaluación de un programa de educación para la salud, otros autores hablan de tres niveles o etapas en los que se puede evaluar: evaluación de proceso, evaluación de impacto y evaluación de resultado. Ver figura 5.

La evaluación de proceso consiste en evaluar la intervención y/o la estrategia educativa. Es en esta etapa se evalúan los materiales y los métodos, las habilidades de los profesionales en la comunicación, y también los aspectos administrativos del desarrollo del programa.

La evaluación de impacto se refiere a los cambios que el programa o estrategia educativa producen en el área del conocimiento, en las actitudes y/o en las conductas en la población. Un ejemplo contundente es la alfabetización.

La evaluación de resultado se ocupa por ejemplo de la morbilidad y de la mortalidad. Es importante resaltar que en intervenciones de educación y promoción para la salud, en general no es posible obtener resultados a corto plazo y que siempre es necesario proyectar a mediano o largo plazo para la espera de los mismos.

Finalmente, toda evaluación debe intentar dar respuestas a las preguntas planteadas de cada aspecto del programa o estrategia educativa, debe poder medir si se han alcanzado las metas propuestas y debe ayudar a determinar si las mismas eran apropiadas.

La evaluación: ¿para quién y por quién?

Figura 5: niveles de evaluación

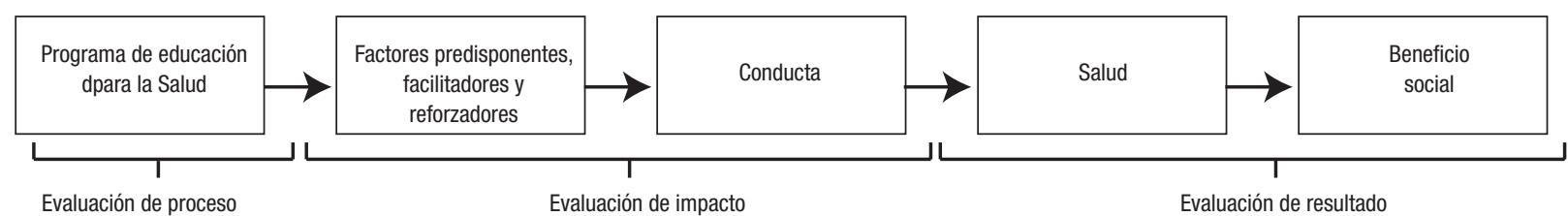

Para responder esta pregunta, a grandes rasgos hacemos referencia a dos tipos de evaluaciones: externas e internas.

1. Evaluaciones externas: están dirigidas por personas ajenas a la organización, al programa y/o a la intervención. En general, este tipo de evaluaciones las suelen realizar las agencias que proveen los fondos o administradores que nada tienen que ver con la planificación y ejecución del programa o estrategia educativa. El plan para tal evaluación viene de afuera y está dirigida por un experto. Con demasiada frecuencia, tanto el proceso como los resultados responden más a las necesidades de los que piden la evaluación que a las necesidades de los que en realidad están participando en el proyecto.

2. Evaluaciones internas: están dirigidas por los participantes del programa. Es interesante e importante en aquellos programas con base comunitaria, que todas las personas involucradas en el proyecto participen de la evaluación, siendo la misma un esfuerzo cooperativo de autocrítica y de reflexión donde, no sólo se tomen en cuenta los resultados de la educación y la acción comunitaria, sino que sea una parte de todo el proceso.

En las evaluaciones suele ser de utilidad solicitar ayuda a personas que estén fuera del programa, con la condición de que comprendan la situación local y que nos ayuden, pero sin hacerse cargo de todo el proceso.

Condiciones para el éxito de una intervención o programa Para que la planificación de una estrategia comunitaria responda a las necesidades reales de la comunidad es indispensable analizar junto con las diversas partes implicadas los problemas que se plantean, los objetivos formulados y la posible elección de una estrategia del siguiente modo: 1) admitiendo ante la comunidad que existe una brecha social y educativa, 2) hacien- do lo necesario para comprender usos, costumbres, creencias y necesidades de la comunidad, 3) tratando de no ser el instructor principal, 4) partiendo de los conocimientos y habilidades que ya tiene la gente y ayudándolos a aprender más, 5) tratando el equipo de salud de hacerse innecesario tan pronto como le sea posible.

Un punto interesante en la planificación desde la comunidad es conocer las redes sociales existentes y si no las hubiere promover precozmente la formación de las mismas. Los recursos comunitarios son valiosos y deben ser cultivados y fortalecidos en forma continua, para poder trabajar participativamente. Para lograr este objetivo es importante que el técnico en salud aúne esfuerzos para insertarse y formar parte de estas redes, logrando de esta manera estimular a sus integrantes y hacer que se consideren miembros activos del proceso (diagnóstico de necesidades, búsqueda de recursos, planificación y ejecución de actividades, gestión, evaluación, etc.). De esta confianza mutua surgirán nuevos conceptos y metodologías científicas que estarán basadas en las preocupaciones diarias y concretas de la comunidad, permitiendo obtener un mayor impacto en los resultados.

Es importante tener presente que va a ser más sencillo trabajar con aquellos grupos que estén en formación o que lleven poco tiempo de funcionamiento, en contraste con hacerlo en un grupo o ámbito comunitario que se desenvuelve más rutinariamente desde hace mucho tiempo. De todas maneras y a diferencia de otras situaciones a las que estamos acostumbrados los médicos, no nos cansaremos de repetir que en este tipo de trabajo es necesario el "tiempo", ya que los cambios suelen ser mucho más lentos.

Los espacios a construir han de imaginarse abiertos, inclusivos, dinámico, cambiantes, plurales, cuestionadores, problematizadores y campo de confrontación de intereses. Por lo tanto no debemos extrañarnos ni desanimarnos ante la aparición de conflicto y/o indignación, o de la necesidad de negociación. 
Por ejemplo, no siempre se debe estar detrás de lograr consensos. Esta búsqueda no debería ser condicionante ni paralizarnos. Los acuerdos no necesariamente hay que imaginarlos absolutos, ni las respuestas únicas y/o definitivas. Es indispensable aprender a construir desde el disenso y aprender a convivir con las diferencias. Es importante tener presente que estos espacios los pueden integrar personas que históricamente están en disputa por situaciones ajenas a nuestros conocimientos pero que no son ajenas a la comunidad que ellos mismos comparten y en la que están inmersos; haciendo que el camino y los espacios que uno proyecte estén en un constante cambio. Por lo tanto, es muy importante que los espacios comunitarios sean dinámicos y abiertos.

\section{Conclusión}

Para que una intervención comunitaria o un programa sean efectivo es importante que incorporen métodos activos de aprendizaje, dirigidos hacia las influencias sociales y a los medios de comunicación de masas y que refuercen los valores individuales y las normas grupales, promoviendo el desarrollo de habilidades, fundamentalmente sociales.

Una estrategia especialmente útil es el trabajo en grupo de iguales. Sus principales ventajas son una mayor adecuación de los contenidos y estrategias, así como una mayor motivación. La persona debe llegar a hacer suya la actividad de aprendizaje (autoaprendizaje) debiendo jugar el educador un papel o rol de facilitador del aprendizaje. Hay que procurar siempre usar métodos participativos que potencien la adquisición de habilidades sociales, la competencia en la comunicación y la resolución de conflictos, que incidan en la responsabilidad, en la autoestima, en la toma de decisiones y que faciliten la práctica de las habilidades aprendidas. En este sentido, vale destacar el mensaje de la Organización Mundial de la Salud (1983): "...si enfocamos la educación sanitaria desde un modelo participativo y adaptado a las necesidades, la población adquirirá una responsabilidad en su aprendizaje y éste no estará centrado en el saber, sino también en el saber hacer". Ver figura 6.

Sólo se construye desde el hacer, desde la reflexión/acción, desde la praxis. Solo el verbo en acción nos permite alcanzar el conocimiento, imaginar respuestas de intervención para nuestros problemas, generar nuevas preguntas y dar perpetuidad y dinamismo al ciclo.

"Es importante que los profesionales de la salud que realizan trabajos comunitarios recuerden que la medición de la eficacia del trabajo comunitario, de la promoción y educación para la salud se parece más a esperar que los cultivos de primavera crezcan después de preparar la tierra en otoño e invierno". Siempre, debe transcurrir un tiempo antes de que los efectos de un entramado de estrategias y procesos de educación coherentes y relacionados comiencen a mostrar resultados que se puedan medir.

En Argentina las actividades de orientación comunitaria en salud han sido poco valoradas por los dirigentes y no han tenido un lugar relevante en las agendas de los profesionales de la salud. Quizás vengan tiempos mejores para "dar poder a la gente" si las organizaciones sanitarias se hacen menos jerárquicas y menos autoritarias...

Figura 6: éxito de un programa de salud comunitaria

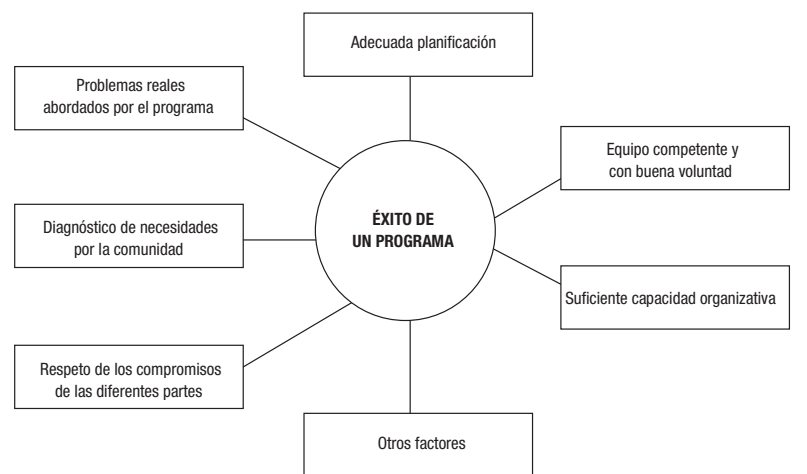

Bibliografía recomendada

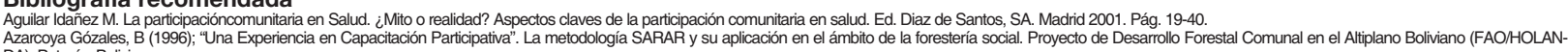
Azarcoya Gózales, B

Bordoni N, Doño R, Misrachi C. Programa de Educación Continua Odontológica No Convencional (PRECONC). Módulo 1: Diagnóstico y educación para la salud. 2edición, 1993. Ed. PALTEX.

Britten N y col. Qualitative research an general practice. British Jorumal of General Practice 1993; 43: 270-271.

Declaración de ALMA-ATA (1978). Conferencia Internacional sobre Atención Primaria de Salud, Alma-Ata, USSR, 6-12 septiembre.

Egg Ezequiel Ander. "¿Qué es el diagnóstico en la Comunidad?" Colección política, servicios y trabajo social. Metodología y práctica del desarrollo de la comunidad. Tomo 1, Editorial Lumen Humanitas.

Egg Ezequiel Ander. "Aspectos operativos y proyectos específicos". Colección polfitica, servicios y trabajo social. Metodología y práctica del desarrollo de la comunidad. Tomo 3 , Editorial Lumen Humanitas.

Egg Ezequiel Ander. "El método del desarrollo de la Comunidad". Colección política, servicios y trabajo social. Metodología y práctica del desarrollo de la comunidad. Tomo 2, Editorial Lumen Humanitas.

Egg Ezequiel Ander. Repensar la investigación-acción participativa. Editorial Lumen.
Fortuny M. Educación para la salud, en: DIVERSOS AUTORES: Iniciativas sociales

Fortuny M. Educación para la salud, en: DIVERSOS AUTORES: Iniciativas sociales de educación informal. Ed. Rialp. Madrid 1990. Pág. 200-249.

Fortuny M. El contenido transversal de la educación para la salud: diseño de la actividad pedagógica. Revista de Ciencias de la Educación, Madrid 1994.

Greene W y col. Educación para la Salud. Ed. Interamericana॰McGraw-Hill. México 1988.
Hawwe P, Degelin D, Hall J. Evaluating Health Promotion. A health Worker's guide. Sydney - Philadelphia - London, $2^{\circ}$ edición, Ed. Maclemnnan+Petty.

Kroeger A y col. Atención Primaria de lsa Salud. Principios y métodos. $2^{\mathrm{a}}$ edición. OPS y Editorial Paz México. México, 1992.

López de Cevallos. Un método para la investigación-acción participativa. Madrid: Popular, 1990.

Marchioni M. Salud, comunidad e intervención comunitaria. En: Enciclopedia profesional de enfermería comunitaria. Ed. McGraw-Hill-Interamericana. Madrid1998.

Polaino-Lorente A. Educación para la salud. Barcelona 1987.

Polaino-Lorente A. Educación para la salud. Barcelona 1987.

San Martín H y Pastor V. Salud comunitaria. Teoría y práctica. Ed. Díaz de Santos, SA. Madrid 1988

Sanchez M. La participación. Metodología y práctica. Madrid: Popular, 1991.

Siles D. Nuevas perspectivas en participación comunitaria: dar poder al paciente. Rev Atención Primaria 1999: 166-168.

Taylor S y col. 1996. "Introducción. Ir hacia la gente". Cap. 1 en Introducción a los métodos cualitativos de investigación. Ed. Paidós. Barcelona. Pág. 15-27.

Técnicas participativas para la educación popular. Alforja, publicaciones de educación popular. $5^{\circ}$ edición CEDEPO. Editorial Humanitas, Buenos Aires

The evidence of Health Promotion Effectiveness. Shaping Public Health in a Neu Europe. A report for the European Commission by the Intemational Union for Health Promotion and Education, 1999

Turabian JL. Apuntes, esquemas y ejemplos de paticipación comuntara Madrid 1902, Ed Díz de Santos.

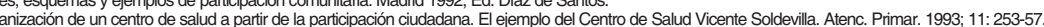

Vuori $\mathrm{H}$ y col. El control de calidad en los servicios sanitarios. Ed. Masson. Barcelona 1991.

Wemer D y Bower B. Aprendiendo a Promover la Salud. $4^{\circ}$ reimpresión. Fundación Hesperian y Centro de estudios educativos, AC. 